\title{
Non-Twist Field Line Mappings for Tokamaks with Reversed Magnetic Shear
}

\author{
M. Roberto ${ }^{1}$, E. C. da Silva ${ }^{2}$, I. L. Caldas ${ }^{2}$, and R. L. Viana ${ }^{3}$ \\ 1. Instituto Tecnológico de Aeronáutica, Centro Técnico Aeroespacial, \\ Departamento de Física, 12228-900, São José dos Campos, SP, Brazil \\ 2. Instituto de Física, Universidade de São Paulo, 05315-970, São Paulo, São Paulo, Brazil \\ 3. Departamento de Física, Universidade Federal do Paraná, 81531-990, Curitiba, PR, Brazil
}

Received on 4 February, 2004; revised version received on 7 June, 2004

\begin{abstract}
The structure of magnetic field lines in a tokamak with reversed magnetic shear is investigated by means of analytically derived area-preserving non-twist Poincaré maps. The basic configuration is the magnetic field produced by an ergodic limiter, superimposed to the tokamak equilibrium field in suitable coordinates. We consider the cases of one and two resonant modes, focusing on magnetic island dimerization and the formation of a transport barrier in the chaotic layer of field lines.
\end{abstract}

\section{Introduction}

The Poincaré map obtained through considering the intersections of the magnetic field line flow - in the equilibrium configuration of a tokamak - with a surface of section, is a nice example of twist map. Since, in the equilibrium MHD approach, magnetic field lines are constrained to lie on flux surfaces, if we use as an action variable any surface label, such as the magnetic flux or even a radial-like coordinate, it turns out that the action variable is constant for successive piercings of the field line with the surface of section. On the other hand, the canonically conjugated angle (which can be a poloidal angle, for example) varies according to the rotational transform of the field lines on that surface. This makes for a twist map, which is the starting point of discrete-time Hamiltonian treatments of magnetic field line structure $[1,2]$. In this way, plasma physicists have the singular opportunity of facing a bona-fide area-preserving canonical map, which comes directly from the physical setting of a problem.

One result which comes from the Hamiltonian treatment of field line flow is the creation of a chaotic magnetic field line layer in the plasma due to the interaction between resonant perturbing fields and the tokamak equilibrium magnetic field. Here the word chaos must be intended in its Lagrangian sense: two field lines, very close to each other, depart exponentially as we follow their revolutions along the toroidal chamber. In terms of the field line map, chaos means an area-filling orbit in the surface of section, through which a field line can wander erratically. This affects in a non-trivial way the transport properties of the tokamak plasma, in a way that is only partially understood at present. Field line chaos can be one of the reasons to explain anomalous transport in tokamaks [3].

The presence of chaotic magnetic field lines in a cer- tain plasma region within the tokamak implies in the loss of the plasma confinement, due to the absence of flux surfaces. Thus we could be mislead to conclude that chaos would be necessarily bad to the fusion program. This is not so, however, because the chaotization of a limited plasma region, if properly handled, can be beneficial to the plasma confinement, as exemplified by the ergodic magnetic limiter [4]. Another situation in which the presence of magnetic field chaos can help plasma confinement is the creation of a transport barrier to reduce particle escape in tokamaks. It has been recently observed that this may occur if there is a negative magnetic shear region within the plasma column $[5,6]$. Such a region is created by means of a non-peaked plasma current density, corresponding to a non-monotonic radial profile for the safety factor [7].

Unlike most area preserving maps used to investigate field line behavior, negative shear configurations are best described by non-twist area-preserving maps[8, 9]. Such maps violate the non-degeneracy condition for the KolmogorovArnold-Moser (KAM) theorem to be valid, so that many well-known results of canonical mappings no longer apply to them $[10,11]$. For example, it may happen that two neighbor island chains approach each other without being destroyed through the breakup of KAM curves [12]. The transport barrier arises from a combination of typical features of non-twist maps: reconnection and bifurcation, occurring in the reversed shear region [13]. This barrier is embedded in a chaotic field line region located in the tokamak peripheral region, and which is generated by an ergodic magnetic limiter [14].

In this paper we will derive non-twist maps by considering the superposition of an ergodic magnetic limiter field on the tokamak equilibrium field with a negative shear region due to a non-peaked plasma current. This map can be used 
to numerically evidence the formation of a transport barrier


Figure 1. (a) Basic geometry of the tokamak; (b) Scheme of an ergodic magnetic limiter.

barrier due to a reconnection-bifurcation mechanism, and its effect on the plasma transport can be inferred from the study of field line diffusion by using the obtained map. Since the chaotic region generated by a limiter reaches the tokamak wall, the transport barrier we obtain is effective for a limited time-span, such that field lines are eventually lost due to radial diffusion and eventual collision with the wall. However, as a consequence of island reconnection and bifurcation, field lines are effectively trapped due to the stickiness effect of the magnetic islands, provided the duration of a discharge is less than the average escape time.

The rest of this paper is organized as follows: in section II we present the equilibrium and perturbing equilibrium fields. Section III shows the area-preserving non-twist map obtained for one and two resonant perturbations, and the field line behavior involving reconnection and bifurcation processes. Our conclusions are left to the last section.

\section{Equilibrium and perturbing mag- netic fields}

We will use a suitable coordinate systems to describe magnetic field line geometry in a tokamak: $\left(r_{t}, \theta_{t}, \varphi_{t}\right)$, given by [15]

$$
\begin{array}{r}
r_{t}=\frac{R_{0}^{\prime}}{\cosh \xi-\cos \omega}, \\
\theta_{t}=\pi-\omega, \quad \varphi_{t}=\Phi
\end{array}
$$

in terms of the more familiar toroidal coordinates $(\xi, \omega, \Phi)$. The meaning of these variables is the following: $r_{t}$ is related to the radial coordinate of a field line with respect to the magnetic axis, which is a circle with radius $R_{0}^{\prime}$ (Fig. 1(a)). $\theta_{t}$ and $\varphi_{t}$ are the poloidal and toroidal angles of this field line, respectively,

The tokamak equilibrium magnetic field $\mathbf{B}^{0}=$ $\left(B_{r_{t}}^{0}, B_{\theta_{t}}^{0}, B_{\varphi_{t}}^{0}\right)$ is obtained from an approximated analytical solution of the equilibrium magneto-hydro-dynamical equation in these coordinates [16]. In order to generate the reversed shear region we have considered a toroidal current density profile with a central hole [17]. There results that the safety factor of the magnetic surfaces, defined as [18]

$$
q\left(r_{t}\right)=\frac{1}{2 \pi} \int_{0}^{2 \pi} \frac{B_{\varphi_{t}}^{0}\left(r_{t}, \theta_{t}\right)}{B_{\theta_{t}}^{0}\left(r_{t}\right)} d \theta_{t}
$$

has a non-monotonic profile. For some values of the safety factor there are two magnetic surfaces with different radii within the plasma column with radius $a$ [17] [see Fig. 2].

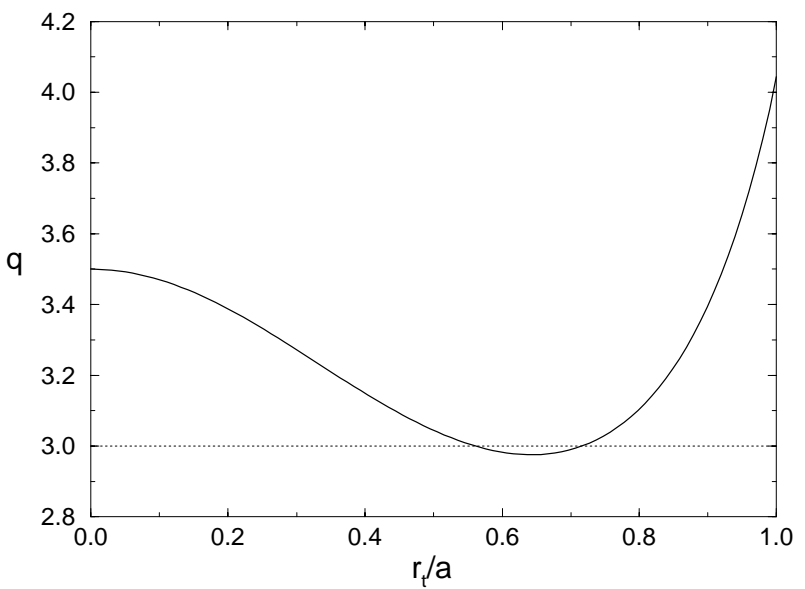

Figure 2. Radial profile of the safety factor for a reversed shear configuration of a tokamak. 
The ergodic magnetic limiter consists of $N_{r}$ current rings of length $\ell$ located symmetrically along the toroidal direction of the tokamak [Fig. 1(b)]. These current rings may be regarded as slices of a pair of external helical windings located at $r_{t}=b$, conducting a current $I_{h}$ in opposite senses for adjacent conductors. The role of these windings is to induce a resonant perturbation in the tokamak, and to achieve this effect we must choose a helical winding with the same pitch as the field lines in the rational surface we want to perturb. This has been carried out by choosing the following winding law [16] $u_{t}=m_{0} \theta_{t}-n_{0} \varphi_{t}=$ constant. In this paper we will consider an ergodic limiter consisting of $N_{r}=4$ rings with mode numbers $\left(m_{0}, n_{0}\right)=(3,1)$ each, carrying a current $I_{h}$.

The magnetic field $\mathbf{B}^{1}$ produced by the resonant helical winding, from which we build the EML rings, is obtained by neglecting the plasma response and the penetration time through the tokamak wall. We were able to obtain an approximated analytical solution in lowest order for the limiter field, through solving the corresponding boundary value problem. The model field to be used in this paper will be the superposition of the equilibrium and limiter fields: $\mathbf{B}=\mathbf{B}^{0}+\mathbf{B}^{1}$.
Since the equilibrium field is axisymmetric, we may set the azimuthal angle, $\varphi_{t}=t$, as a time-like variable, and put the magnetic field line equations in a Hamiltonian form

[19]

$$
\begin{aligned}
\frac{d \mathcal{J}}{d t} & =-\frac{\partial H}{\partial \vartheta}, \\
\frac{d \vartheta}{d t} & =\frac{\partial H}{\partial \mathcal{J}}
\end{aligned}
$$

where $(\mathcal{J}, \vartheta)$ are the action-angle variables of a Hamiltonian $H$, its explicit form being given in Ref. [20]. In that paper we analyzed exact but numerically obtained maps, whereas in this work we derive analytical, albeit approximate, forms of these maps.

The addition of the magnetic field produced by a resonant helical winding may be regarded as a Hamiltonian perturbation. In order to include the effect of the finite length $\ell$ of each EML ring, which is typically a small fraction of the total toroidal circumference $2 \pi R_{0}^{\prime}$, we model its effect as a sequence of delta-functions centered at each ring position. The Hamiltonian for the system is thus

$$
H(\mathcal{J}, \vartheta, t)=H_{0}(\mathcal{J})+\frac{\ell}{R_{0}^{\prime}} H_{1}(\mathcal{J}, \vartheta, t) \sum_{k=-\infty}^{+\infty} \delta\left(t-k \frac{2 \pi}{N_{r}}\right),
$$

We consider the perturbing Hamiltonian as a periodic function in both $\vartheta$ and $t$ variables, such that it can be written as a Fourier series in these variables. There results a model Hamiltonian

$$
H(\mathcal{J}, \vartheta, t)=H_{0}(\mathcal{J})+\epsilon \sum_{m=0}^{2 m_{0}} H_{m}^{*}(\mathcal{J}) \cos \left(m \vartheta-n_{0} t\right) \sum_{k=-\infty}^{+\infty} \delta\left(t-k \frac{2 \pi}{N_{r}}\right)
$$

where $H_{m}^{*}$ are Fourier coefficients, whose detailed forms can be found in Ref. [20], and the perturbation strength is expressed by the parameter

$$
\epsilon=-2\left(\frac{\ell}{2 \pi R_{0}^{\prime}}\right)\left(\frac{I_{h}}{I_{e}}\right)
$$

which is usually small, since in experiments we have $\ell \ll 2 \pi R_{0}^{\prime}$ and $I_{h} \ll I_{e}$, where $I_{e}$ is the coil current producing the toroidal equilibrium field.

\section{Non-twist maps for one and two re- sonant modes}

The non-monotonicity of the radial safety factor profile makes possible to have two resonant surfaces with the same value of the safety factor. From the Hamiltonian theory of quasi-integrable systems we know that there appear two island chains centered at these radii. A periodic island results from the resonance between the equilibrium tokamak field and one mode of the perturbation field. Let us suppose that we are dealing with a resonant perturbation with (positive integer) mode numbers $\left(m_{0}, n_{0}\right)$, such that the safety factor $m_{0} / n_{0}$ is a rational number. In terms of the action-angle variables of the unperturbed Hamiltonian, corresponding to the tokamak equilibrium field, this is a frequency, which is the same for the two island chains centered at $\mathcal{J}_{1}^{*}$ and $\mathcal{J}_{2}^{*}$

$$
\omega_{0}\left(\mathcal{J}_{1}^{*}\right)=\omega_{0}\left(\mathcal{J}_{2}^{*}\right)=\frac{\partial H_{0}}{\partial \mathcal{J}}=\frac{n_{0}}{m_{0}}
$$

The dynamical behavior in the vicinity of the resonant surfaces located at $\mathcal{J}_{1}^{*}$ and $\mathcal{J}_{2}^{*}$ can be investigated by picking up from the model Hamiltonian, Eq. (7), the resonant term corresponding to the frequency $n_{0} / m_{0}$ :

$$
H(\mathcal{J}, \vartheta, t)=H_{0}(\mathcal{J})+\epsilon H_{m_{0}}^{*}(\mathcal{J}) \cos \left(m_{0} \vartheta-n_{0} t\right) \sum_{k=-\infty}^{+\infty} \delta\left(t-k \frac{2 \pi}{N_{r}}\right)
$$


and expanding the Hamiltonian in the neighborhood of the rational surface beyond the cubic approximation. This is actually necessary for taking into account the fact that the unperturbed Hamiltonian $H_{0}$ fails to satisfy KAM theorem, due to the non-monotonicity of the frequency. As a conse- quence, the island chains are not pendular (i.e., their widths do not increase as the square root of $\epsilon$ ), and we need a higher order approximation in order to capture the essentials of the twins islands' behavior.

After dropping the constant terms, we obtain

$$
\begin{aligned}
H(\Delta \mathcal{J}, \vartheta, t) & \approx \omega_{0}\left(\mathcal{J}^{*}\right) \Delta \mathcal{J}+\left.\frac{1}{2} \frac{d \omega_{0}}{d \mathcal{J}}\right|_{\mathcal{J}=\mathcal{J}^{*}}(\Delta \mathcal{J})^{2}+\left.\frac{1}{6} \frac{d^{2} \omega_{0}}{d \mathcal{J}^{2}}\right|_{\mathcal{J}=\mathcal{J}^{*}}(\Delta \mathcal{J})^{3}+\cdots \\
& +\epsilon H_{m_{0}}^{*}\left(\mathcal{J}^{*}\right) \cos \left(m_{0} \vartheta-n_{0} t\right) \sum_{k=-\infty}^{+\infty} \delta\left(t-k \frac{2 \pi}{N_{r}}\right)
\end{aligned}
$$

where $\Delta \mathcal{J}=\mathcal{J}-\mathcal{J}^{*}$.

We can simplify this expression by performing a canonical transformation to new action-angle variables $\left(\mathcal{J}^{\prime}, \vartheta^{\prime}\right)$ which eliminates the explicit time-dependence, what can be done through the generating function

$$
S\left(\mathcal{J}^{\prime}, \vartheta, t\right)=\left(\vartheta-\frac{n_{0}}{m_{0}} t\right) \mathcal{J}^{\prime}
$$

leading to the local Hamiltonian

$$
\begin{gathered}
H^{\prime}\left(\mathcal{J}^{\prime}, \vartheta^{\prime}\right)=\frac{M}{2} \mathcal{J}^{\prime 2}-\frac{W}{3} \mathcal{J}^{\prime 3} \\
+K \cos \left(m_{0} \vartheta^{\prime}\right) \sum_{k=-\infty}^{+\infty} \delta\left(t-k \frac{2 \pi}{N_{r}}\right),
\end{gathered}
$$

in which we have defined

$$
\begin{aligned}
M\left(\mathcal{J}^{*}\right) & \left.\equiv \frac{d \omega_{0}}{d \mathcal{J}}\right|_{\mathcal{J}=\mathcal{J}^{*}}, \\
W\left(\mathcal{J}^{*}\right) & \left.\equiv \frac{1}{2} \frac{d^{2} \omega_{0}}{d \mathcal{J}^{2}}\right|_{\mathcal{J}=\mathcal{J}^{*}}, \\
K\left(\mathcal{J}^{*}\right) & \equiv \epsilon H_{m_{0}}^{*}\left(\mathcal{J}_{1,2}^{*}\right) .
\end{aligned}
$$

After dividing both sides of Eq. (13) by $M$, what amounts to re-scale the field line Hamiltonian, we have

$$
\begin{gathered}
H\left(\mathcal{J}^{\prime}, \vartheta^{\prime}\right)=\frac{1}{2} \mathcal{J}^{\prime 2}-\frac{\alpha}{3} \mathcal{J}^{3} \\
+\kappa \cos \left(m_{0} \vartheta^{\prime}\right) \sum_{k=-\infty}^{+\infty} \delta\left(t-k \frac{2 \pi}{N_{r}}\right),
\end{gathered}
$$

where $\alpha=W / M$ and $\kappa=K / M$.

In the $\alpha=0$ limit, the Hamiltonian above reduces to that of a nonlinear pendulum, which is the standard procedure used in perturbation theory to describe the phase-space structure near a given resonance [2]. Hence, $\alpha$ measures, so as to speak, the non-pendular character of the island chains. The corresponding Hamilton equations are

$$
\begin{aligned}
\frac{d \mathcal{J}^{\prime}}{d t} & =m_{0} \kappa \sin \left(m_{0} \vartheta^{\prime}\right) \sum_{k=-\infty}^{+\infty} \delta\left(t-k \frac{2 \pi}{N_{r}}\right), \\
\frac{d \vartheta^{\prime}}{d t} & =\mathcal{J}^{\prime}\left(1-\alpha \mathcal{J}^{\prime}\right) .
\end{aligned}
$$

Writing down the summation in $t$ as a Fourier series and isolating the resonant term, we can use these equations to predict the widths of each non-pendular island as well as to make an estimation of the critical value of the limiter current necessary to their reconnection [20].

The fact that the "time"-dependence of the Hamilton equations is in the form of a periodic sequence of delta function kicks enables us to define discretized variables [21]

$$
\begin{aligned}
\vartheta_{k}^{\prime} & =\lim _{\epsilon \rightarrow 0} \vartheta^{\prime}\left(t=t_{k}+\epsilon\right) \\
\mathcal{J}_{k}^{\prime} & =\lim _{\epsilon \rightarrow 0} \mathcal{J}^{\prime}\left(t=t_{k}+\epsilon\right)
\end{aligned}
$$

as the angle and action, respectively, just after the k-th kick, which occurs at time $t_{k}=k\left(2 \pi / N_{r}\right)$ (note that one Poincaré section of the field line flow is done after $N_{r} m_{0}$ turns around the torus, rather than simply sampling out the field line position after each turn, as usually done). The resulting field line map is thus

$$
\begin{aligned}
\mathcal{J}_{k+1}^{\prime} & =\mathcal{J}_{k}^{\prime}+m_{0} \kappa \sin \left(m_{0} \vartheta_{k+1}^{\prime}\right), \\
t_{k+1} & =t_{k}+\frac{2 \pi}{N_{r}} \\
\vartheta_{k+1}^{\prime} & =\vartheta_{k}^{\prime}+\frac{2 \pi}{N_{r}} \mathcal{J}_{k}^{\prime}\left(1-\alpha \mathcal{J}_{k}^{\prime}\right) \quad(\bmod 2 \pi) .
\end{aligned}
$$

If we switch off the perturbation $(\kappa=0)$, the map above reduces to a simple non-twist map, for which all trajectories are bound to invariant tori with constant $\mathcal{J}^{\prime}$. The nonmonotonic character of the equilibrium field is expressed in the $\alpha$ term which appears in the winding number term in Eq. 24. From now on we choose an ergodic limiter with $N_{r}=4$ rings, uniformly spaced from each other along the tokamak torus (i.e. with angular separation $\pi / 2$ ), each of them with $m_{0}=3$ pairs of wires. The plasma current density profile is supposed to yield a non-monotonic profile for the winding number $\omega=\partial H_{0}\left(\mathcal{J}^{\prime}\right) / \partial \mathcal{J}^{\prime}$, such that there are two values of the action variable for $\omega=1 / 3$.

If we pick up two resonant modes from the model Hamiltonian (7), there will appear terms in $\cos \left[m_{0} \vartheta^{\prime}-\right.$ $\left.\left(n_{0} / m_{0}\right) t\right]$ and $\cos \left[\left(m_{0}+1\right) \vartheta^{\prime}-\left(n_{0} / m_{0}\right) t\right]$. The derivation of the field line map is similar to that described in the previous section, and results in the following system of equations 


$$
\begin{aligned}
\mathcal{J}_{k+1}^{\prime} & =\mathcal{J}_{k}^{\prime}+m_{0} \kappa \sin \left(m_{0} \vartheta_{k+1}^{\prime}\right)+\left(m_{0}+1\right) \tilde{\kappa} \sin \left[\left(m_{0}+1\right) \vartheta_{k+1}^{\prime}\right] \\
\vartheta_{k+1}^{\prime} & =\vartheta_{k}^{\prime}+\frac{2 \pi}{N_{r}} \mathcal{J}_{k}^{\prime}\left(1-\alpha \mathcal{J}_{k}^{\prime}\right) \quad(\bmod 2 \pi) \\
t_{k+1} & =t_{k}+\frac{2 \pi}{N_{r}}
\end{aligned}
$$

where $\tilde{\kappa}=\kappa_{1} I_{h}, \kappa_{1}$ being the amplitude of the second resonant mode. The map is obtained for each $m_{0}$ turns around the tokamak torus.

For small limiter currents [Fig. 3(a)] we have: (i) two chains of non-pendular islands centered at the points $\mathcal{J}_{1}^{*}$ and $\mathcal{J}_{2}^{*}$, due to the non-monotonic character of the safety factor profile [see Fig. 2]; (ii) a third chain of pendular islands centered at the position of the second resonance $\left(m_{0}+1\right) / n_{0}=4 / 1$, since for this value of $q$ we have just one resonant surface, according to Fig. 2.

In the region of reversed magnetic shear, due to the locally non-KAM nature of the Hamiltonian, the map is nontwist. Hence, the two non-pendular islands of the 3/1-chain can approach each other without destruction of tori. For a perturbation strong enough [Fig. 3(b)] the island chains dimerize, forming a unique sequence of islands which share a common separatrix. This is called reconnection, but it is rather a topological effect, involving no dissipative breakup and re-wiring of the field lines, as it occurs in astrophysical and fusion situations. After the reconnection takes place we find curves (meanders) which encircle both sets of islands, forming a unique path which will be the foundation of the transport barrier to be described.

The action of an ergodic limiter actually produces an infinite number of resonant modes due to the localized nature of the generated fields in the toroidal direction (note the infinite terms in the summation in Eq.(17), for example). Since the magnetic field generated by a magnetic limiter falls off rapidly as we move apart from the tokamak wall [16], the amplitudes of the corresponding resonant modes also decrease as we go into the tokamak center. Hence, the main resonance is just that produced near the tokamak wall, the others having exponentially smaller widths.

In fact, as the limiter current $I_{h}$ increases, all mode amplitudes are augmented, yet not in the same proportion. For many purposes these higher modes are not important and hence it suffices to pick up just one resonant mode from the field line Hamiltonian (17). However, in order to study the formation of a large chaotic region inside the tokamak plasma, it is necessary to take into account a second resonant mode from the infinite number of possible modes. Since this second chain is now pendular, they will not dimerize with the first resonant mode. As the perturbation strength increases both islands become wider and they interact as predicted by the global stochasticity mechanisms widely studied in the literature [Fig. 3(c)] [2].
As the limiter current increases further [Fig. 3(c)] we can see that: (a) the twin chains of the first mode have already dimerized and suffered reconnection, with open surfaces exploring the former twin chains (this is actually necessary for the transport barrier be present); and (b) there are visible chaotic layers surrounding both sets of islands. However, as the limiter current is still not high enough, there are many surviving invariant curves between the sets of islands. This is, in fact, a result from KAM theorem which applies here because this region has a monotonic frequency profile and, at least locally, the conditions for the KAM theorem to be valid are fulfilled.

Even higher limiter currents will destroy entirely the invariant curves between both chains and a wide chaotic layer appears surrounding both modes. At least in principle, Chirikov's criterion for global stochasticity applies in this case and predicts the formation of such layer [1]. The transport barrier appears embedded in this large chaotic layer and is structured around the path of open curves which encircled the formerly dimerized islands after reconnection. This layer acts as an effective transport barrier since it hampers the radial diffusion of field lines, and arises from the "stickiness" effect that the region near the former separatrices of islands exert on the chaotic trajectories. This effect has been related to manifold reconnection in Ref. [13] occurring inside the chaotic region. This barrier is effective provided the duration of a typical tokamak plasma discharge is less than the average escape time.

\section{Conclusions}

We used analytically obtained non-twist field line maps to study the effects of an ergodic magnetic limiter on the magnetic field line structure which results from a plasma current profile exhibiting reversed magnetic shear. The non-twist nature of the map allows island chains to approach and dimerize, as the limiter current builds up, in a reconnective process. In the case of one resonant mode, although there is already a symmetry-breaking cause of non-integrability, the chaotic field line region is too narrow. The creation of a reasonably wide chaotic layer is only feasible when considering two resonant modes. The second mode interacts with the dimerized island chains created by the first mode in a conventional way, predicted by global stochasticity mechanisms, eventually forming a chaotic region. 

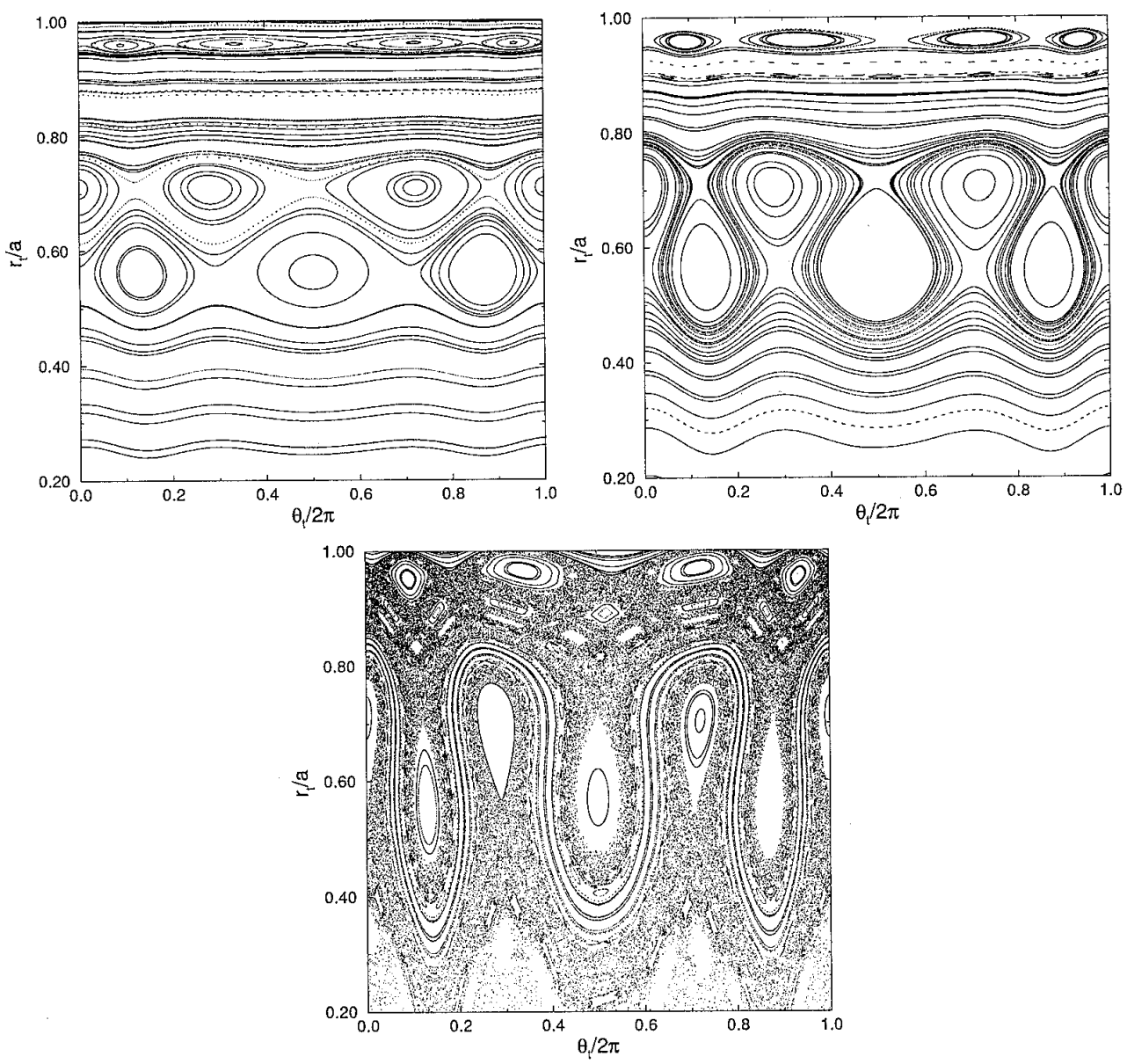

Figure 3. Poincaré maps (in scaled toroidal coordinates) for two resonant modes with (a) $\kappa_{0}=-6.33101 \times 10^{-6}, \kappa_{1}=2.91385 \times 10^{-6}$; (b) $\kappa_{0}=-1.477235 \times 10^{-5}, \kappa_{1}=6.798983 \times 10^{-6}$; (c) $\kappa_{0}=-8.441344 \times 10^{-5}, \kappa_{1}=3.885133 \times 10^{-5}$.

This layer works like an effective transport barrier, with respect to the typical plasma duration. The trapping is more intense around the shearless plasma, and results from the properties of field line trajectories in the vicinity of separatrices of islands bordering the chaotic region. This transport barrier can help plasma confinement preventing energetic charged particles from escaping out radially to an eventual collision with the tokamak inner wall.

\section{Acknowledgments}

This work was made possible with partial financial support of the following agencies: CNPq and FAPESP.

\section{References}

[1] B. Chirikov, Phys. Reports (1979)

[2] A. J. Lichtenberg and M. A. Lieberman, Regular and Chaotic Dynamics, 2nd. Edition (Springer-Verlag, New York-BerlinHeidelberg, 1992).

[3] J. D. Meiss, Rev. Mod. Phys. 64, 796 (1992). Neoclassical transport, (North Holland Publ., Amsterdam, 1988).
[4] J. E. S. Portela, R. L. Viana, and I. L. Caldas, Physica A 317, 411 (2003).

[5] F. M. Levinton, M. C. Zarnstoff, S. H. Batha, et al., Phys. Rev. Lett. 75, 4417 (1995).

[6] E. J. Strait, L. L. Lao, M. E. Manel, et al., Phys. Rev. Lett. 75, 4421 (1995).

[7] E. Mazzucato, S. H. Batha, M. Beer, et al., Phys. Rev. Lett. 77, 3145 (1996).

[8] P. J. Morrison, Phys. Plas. 7, 2279 (2000).

[9] R. Balescu, Phys. Rev. E 58, 3781 (1998).

[10] D. Castillo-Negrete, J. M. Greene, and P. J. Morrison, Physica D 91, 1 (1996).

[11] E. Petrisor, J. H. Misguich, and D. Constantinescu, Chaos, Solit. \& Fract. 18, 1085 (2003).

[12] J. E. Howard and S. M. Hohs, Phys. Rev. A 29, 418 (1984).

[13] G. Corso, F. B. Rizzato, Phys. Rev. E 58, 8013 (1998).

[14] E. C. da Silva, I. L. Caldas, and R. L. Viana, Phys. Plasmas 8, 2855 (2001); Chaos, Solit. \& Fract. 14, 403 (2002).

[15] M. Y. Kucinski, I. L. Caldas, L. H. A. Monteiro, and V. Okano, J. Plas. Phys. 44, 303 (1990). 
[16] E. C. da Silva, I. L. Caldas, and R. L. Viana, IEEE Trans. Plas. Sci. 29, 617 (2001).

[17] G. A. Oda and I. L. Caldas, Chaos, Solit. \& Fract. 5, 15 (1995); G. Corso, G. A. Oda, and I. L. Caldas, Chaos, Solit. \& Fract. 8, 1891 (1997).

[18] J. Wesson, Tokamaks (Oxford University Press, 1987).
[19] I. L. Caldas, R. L. Viana, M. S. T. Araujo, et al., Braz. J. Phys. 32, 980 (2002).

[20] M. Roberto, E. C. da Silva, I. L. Caldas, and R. L. Viana, Phys. Plasmas 11, 214 (2004).

[21] E. Ott, Chaos in Dynamical Systems (Cambridge University Press, Cambridge, 1994) 